\title{
CONTRATACIÓN ADMINISTRATIVA Y ADMINISTRACIÓN LOCAL EN LA AUTORIDAD VASCA DE LA COMPETENCIA. EL CASO PINOSOLO ${ }^{1}$
}

\author{
Guillermo Aranzabe Pablos
}

\begin{abstract}
Sumario: 1. Introducción. 2. Antecedentes del CASO. 3. Concurso PÚBLICO DEL «COMPLEJO DEPORTIVO PINOSOLO». 4. DENUNCIA. 5. RESOLUCIÓN DEL CONSEJO VASCO DE LA COMPETENCIA (CVC). 5.1. Prueba de presunciones. 5.1.1. Similitud de planos. 5.1.2. Similitud de archivos. 5.1.3. Facturas emitidas por PUJOL. 5.2. Subsunción de las conductas en el artículo 1.1 de la LDC. 5.2.1 Existencia de una concertación. 5.2.2 Existencia de un falseamiento de la competencia en el mercado. 5.2.3 Conclusión. 5.3. Subsunción de las conductas en el artículo 3 de la LDC. 5.3.1. El comportamiento de las empresas como acto de competencia desleal. 5.3.2. Falseamiento de la competencia. 5.3.3. Afectación al interés público. 5.3.4 Conclusión. 5.4. Concurrencia de infracciones. 5.5. Sanción. 6. CONSIDERACIONES FINALES.
\end{abstract}

\section{INTRODUCCIÓN}

Este trabajo analiza un caso examinado en la Autoridad Vasca de la Competencia por infracción a la normativa reguladora de la defensa de la competencia.

${ }^{1}$ Resolución CVC 05/2012, asunto CONCURSO PINOSOLO. http://www.competencia.euskadi.net/contenidos/informacion/resoluciones/es_resoluci/adjuntos/concurso $\% 20$ pinosolo $\% 20$ pdd.pdf 
El Consejo Vasco de la Competencia (en adelante, $\mathrm{CVC})^{2}$ ha considerado probado en su Resolución de 21 de mayo de 2013 que esta infracción se cometió en el seno de una licitación pública convocada por una administración local para la construcción de una infraestructura deportiva ${ }^{3}$.

Me propongo, por tanto, exponer los elementos relevantes del caso y explicitar los argumentos utilizados por el Consejo Vasco de la Competencia para declarar probada la comisión de la infracción.

\section{ANTECEDENTES DEL CASO}

El 26 de diciembre de 2006, el Ayuntamiento de Leioa (Bizkaia) adjudicó una licitación pública referente al contrato de redacción de proyectos y dirección de obras de urbanización en el municipio de Leioa. La adjudicación se hizo a favor de la empresa ARCAIN INGENIERIA y ARQUITECTURA S.L. (en adelante, ARCAIN) y otras dos empresas más.

La adjudicación del referido contrato implicaba que Ayuntamiento de Leioa encargaría una serie de labores de consultoría y asesoramiento a ARCAIN en relación a tres actuaciones urbanísticas a realizar en el área de Pinosolo-Torresolo: un polideportivo denominado «Complejo Deportivo Pinosolo», una instalación de ola artificial y una zona lúdico-deportiva exterior. Las encomiendas se separaron en el tiempo y fueron de diferente entidad.

Así, respecto del concurso del «Complejo Deportivo Pinosolo» se le encomendó la consultoría y asistencia técnica en la ejecución de labores previas (tales como trabajos de campo, estudio de necesidades y fórmulas de contratación, elaboración de los pliegos de prescripciones técnicas), así como se le hizo parte de la Comisión Evaluadora que prestaría asistencia técnica al Consejo de Administración de LEIOA KIROLAK S.A.U. (en adelante, LEIOA KIROLAK $)^{4}$ en la adjudicación del concurso del «Complejo Deportivo Pinoso-

2 Órgano resolutor de la Autoridad Vasca de la Competencia. Está compuesto por las siguientes personas: Dña. María Pilar Canedo Arrillaga, Presidente; Dña. Natividad Goñi Urriza, Vocal; y D. Rafael Iturriaga Nieva, Vocal.

${ }^{3}$ La Resolución en cuestión ha sido objeto de recurso contencioso-administrativo ante el Tribunal Superior de Justicia del País Vasco, no habiéndose emitido pronunciamiento judicial hasta la fecha.

${ }^{4}$ Sociedad mercantil unipersonal de titularidad del Ayuntamiento de Leioa, cuyo objeto social consiste en realizar estudios, proyectar, construir, conservar, mantener, financiar y explotar, por si misma o por terceros, las instalaciones deportivas que se le encomiendan por el Ayuntamiento.

Fue constituida el 16 de julio de 2008 en el momento de abordar el desarrollo urbanístico de la zona de Pinosolo-Torresolo en lo que se refiere a la proyección y edificación de los equipamientos deportivos previstos. 
lo», lo que supone asignar a ARCAIN dos de los tres miembros de la Comisión Evaluadora (55\% de la puntuación total de los criterios de adjudicación).

Respecto de la Instalación «Ola Artificial», se encomendó a ARCAIN la redacción del proyecto y dirección de la obra. Asimismo, respecto de la «Zona lúdico-deportiva» exterior, se encomendó a ARCAIN la redacción del proyecto básico y la dirección de la obra.

\section{CONCURSO PÚBLICO DEL «COMPLEJO DEPORTIVO PINOSOLO»}

LEIOA KIROLAK convocó el 29 de agosto de 2009 la licitación pública para la redacción de proyecto, dirección de obra, coordinación en materia de seguridad y salud y ejecución de las obras del «Complejo Deportivo Pinosolo» con un presupuesto de 50.191.363,50 euros.

El Consejo de Administración de LEIOA KIROLAK acordó recabar el asesoramiento técnico que considerase oportuno para informar las ofertas del concurso del «Complejo Deportivo Pinosolo». Se acordó crear una Comisión Evaluadora y, para constituirla, se contrataron los servicios de una empresa experta en lo relativo a la ingeniería y la arquitectura, así como los servicios de un experto en gestión y funcionalidad. Esas empresas expertas resultaron ser ARCAIN (que, en su labor de consultoría, aportó dos técnicos) y AFP GRUPO (que aportó uno).

El 8 de febrero de 2010 la Comisión Evaluadora emitió su propuesta de adjudicación del concurso «Complejo Deportivo Pinosolo». El resultado emitido por la Comisión situaba en primer lugar por puntuación total a la UTE Aros-Viuda De SAINZ.

El 10 de febrero de 2010 el Consejo de Administración de LEIOA KIROLAK aprobó el acuerdo sobre la adjudicación provisional del concurso del «Complejo Deportivo Pinosolo» a favor de la Ute Aros-Viuda De SAinz. Contra dicha adjudicación provisional DGM DE ARQUITECTOS, S.L.P., empresa de arquitectura participante en otra UTE (en adelante, DGM), interpuso recurso especial al amparo del artículo 37 de la Ley 30/2007, de 30 de octubre, de Contratos del Sector Público (LCSP) ${ }^{5}$. Dicho recurso fue estimado parcial-

5 «1. Las decisiones a que se refiere el apartado 2 del presente artículo que se adopten en los procedimientos de adjudicación de contratos sujetos a regulación armonizada, incluidos los contratos subvencionados, contratos de servicios comprendidos en las categorías 17 a 27 del Anexo II de cuantía igual o superior a 211.000 euros, o contratos de gestión de servicios públicos en los que el presupuesto de gastos de primer establecimiento sea superior a 500.000 euros y el plazo de duración superior a cinco años, deberán ser objeto del recurso especial en materia de contratación que se regula en este artículo con anterioridad a la interposición del recurso contencioso administrativo, sin que proceda la interposición de recursos administrativos ordinarios contra los mismos. No se dará este recurso en relación con los actos dictados en procedimientos de adjudicación que se sigan por el trámite de emergencia regulado en el artículo 97. 
mente el 28 de junio de 2010, anulándose la adjudicación provisional para que, retrotrayendo actuaciones, se procediese a una nueva valoración.

El 15 de julio de 2010 la Comisión Evaluadora emitió un segundo informe de evaluación de las ofertas. En el informe figuraba de nuevo en primer lugar por puntuación total la Ute Aros-ViUdA De SAINZ.

El 20 de julio de 2010 el Consejo de Administración de LEIOA KIROLAK aprobó el acuerdo sobre nueva adjudicación provisional del concurso «Complejo Deportivo Pinosolo» a favor de la Ute Aros-Viuda De Sainz.

Contra esta segunda adjudicación provisional DGM interpuso recurso especial al amparo del citado artículo 37 de la LCSP. Dicho recurso fue estimado parcialmente el 12 de noviembre de 2010, anulándose nuevamente la adjudicación provisional. A juicio del Consejo de Administración de LEIOA KIROLAK, no había estado completamente garantizada la imparcialidad y transparencia de la valoración realizada para acordar la adjudicación provisional. Por ello, se estableció de nuevo la obligación de retrotraer las actuaciones y realizar una nueva valoración por una Comisión Evaluadora diferente.

El 23 de diciembre de 2010, por Resolución del Presidente del Consejo de Administración de LEIOA KIROLAK, se designó la segunda Comisión Evaluadora para el concurso del «Complejo Deportivo Pinosolo». La nueva Comisión estuvo formada por un representante de cada una de las siguientes empresas: BM² Arquitectos, S.C.-MnB; AbC-DeF; E IdeA \& Dimensiona, SLP.

El 11 de febrero de 2011, el Consejo de Administración de LEIOA KIROLAK aprobó el acuerdo sobre la adjudicación definitiva del concurso del «Complejo Deportivo Pinosolo» a favor de la Ute Aros-Viuda De SAinz.

En julio de 2012, el Ayuntamiento de Leioa renunció a la construcción del «Complejo Deportivo Pinosolo» y rescindió el contrato suscrito con la Ute Aros-Viuda De Sainz.

\section{DENUNCIA}

El 24 de enero de 2011 DGM presentó denuncia ante el Servicio Vasco de Defensa de la Competencia ${ }^{6}$. En la denuncia se alegó que se habría producido

2. Serán susceptibles de recurso especial los acuerdos de adjudicación provisional, los pliegos reguladores de la licitación y los que establezcan las características de la prestación, y los actos de trámite adoptados en el procedimiento antecedente, siempre que éstos últimos decidan directa o indirectamente sobre la adjudicación, determinen la imposibilidad de continuar el procedimiento o produzcan indefensión o perjuicio irreparable a derechos o intereses legítimos.

(...)»

6 Antiguo órgano instructor en materia de defensa de la competencia de la Comunidad Autónoma de Euskadi. Extinguido en virtud de lo dispuesto en la Disposición Adicional segunda de la Ley 1/2012, de 2 de febrero, de la Autoridad Vasca de la Competencia. 
un intercambio de información entre Arcain y la Ute Aros-ViUda De SaInZ, de la que formaban parte Aros Estudio De ArQuitectura, S.A. (en adelante, Aros) y Excavaciones Viuda De Sainz, S.A. (en adelante Viuda De Sainz).

A juicio del denunciante, se habría dado una concertación entre ARCAIN y la Ute Aros-Viuda De Sainz en la que el redactor del proyecto, Pujol arQUiTECTURA, S.L.P. (en adelante PuJOL), habría jugado un papel decisivo. La concertación tendría como objetivo la adjudicación del concurso del «Complejo Deportivo Pinosolo» a la Ute Aros-VIUdA De SaInZ y se habría instrumentado mediante tres elementos: un intercambio de información previa sobre los pliegos del concurso y sobre la oferta de la UTE AROS-VIUDA DE SAINZ; una sobrevaloración de la oferta de la UtE Aros-ViUdA De SAINZ; y una infravaloración de la oferta de la UTE del denunciante.

Con relación al intercambio de información el denunciante aportó como prueba un CD (en adelante CD1) que recogía el proyecto técnico que ARCAIN había realizado para la actuación urbanística de la Instalación «Ola Artificial». El CD1 contenía, junto con la información referida a la Instalación «Ola Artificial» que debía hacerse disponible para todos los licitadores en el concurso del «Complejo Deportivo Pinosolo» (en aras de conseguir coherencia entre los proyectos), un plano de las cubiertas que correspondía precisamente a la actuación urbanística en el «Complejo Deportivo Pinosolo».

Ese plano (que según informe técnico presentado por la denunciante provendría del proyecto técnico redactado por PUJOL) se presentó posteriormente por la Ute ARos-VIUdA De SAINZ a la licitación del «Complejo Deportivo Pinosolo». Es decir, según el denunciante, se le habrían entregado los planos de la UTE que resultaría adjudicataria de la licitación del «Complejo Deportivo Pinosolo» en 2009, durante la fase de preparación del proceso, por parte de la empresa pública encargada de la gestión del proyecto de la Instalación «Ola Artificial», la Sociedad Pública Foral KirolgintZAN S.A.7 (en la actualidad, AZPIEGITURAK, S.A. ${ }^{8}$ ) (en adelante KIROLGINTZAN).

Por ello, el denunciante sostuvo que la oferta de la Ute Aros-VIUDA DE SAINZ no fue secreta, tal y como exige el artículo 129.2 de la LCSP, vigente

${ }^{7}$ Sociedad constituida en el año 2001, bajo la denominación social de Meaztegi, S.A.U. Posteriormente, en abril de 2006, se cambió la denominación por Kirolgintzan, S.A.U. Tenía como objeto social la planificación, elaboración de proyectos, construcción, conservación, mantenimiento y explotación, de cualquier infraestructura, equipamientos, instalaciones, servicios, complejos edificatorios o bienes destinados a cualquier actividad de carácter deportivo, de esparcimiento o cultural, derivados de acuerdos adoptados por la Diputación Foral de Bizkaia. Se le encargó la gestión del proyecto denominado «Instalación de ola artificial».

8 Sociedad Pública Unipersonal de la Diputación Foral de Bizkaia, creada el 6 de julio de 2010 fruto de la fusión de las empresas forales Azpiegitura, S.A.U., Bideak-Bizkaiko Bideak, S.A.U., Kirolgintzan S.A.U y Bizkailur, S.A.U. 
en el momento en el que ocurrieron los hechos objeto de denuncia'. El denunciante estimó, además, que el acuerdo entre los operadores era apto para restringir la competencia en el citado concurso público.

La denuncia por infracción de la normativa de competencia se presentó contra las mercantiles ArCain, Aros, Viuda De Sainz y Pujol.

\section{RESOLUCIÓN DEL CONSEJO VASCO DE LA COMPETENCIA (CVC)}

\subsection{Prueba de presunciones}

Para acreditar la existencia de infracción a la normativa de la competencia el CVC empleó la prueba de presunciones basada en los hechos acreditados como probados en el Pliego de Concreción de Hechos emitido por el instructor del expediente sancionador ${ }^{10}$.

\subsubsection{Similitud de planos}

En 2008 el Ayuntamiento de Leioa adjudicó a ARCAIN la redacción del proyecto y dirección de obra de la Instalación «Ola Artificial». Como el proyecto del «Complejo Deportivo Pinosolo» se complementaba con la Instalación «Ola Artificial», se concedió acceso al proyecto de Instalación «Ola Artificial», elaborado por ARCAIN, a todas las empresas que mostraron su intención de presentar ofertas en dicho concurso.

KIROLGINTZAN, en cumplimiento de sus funciones de gestión en el proyecto de la Instalación «Ola Artificial», fue la empresa encargada de entregar

${ }^{9}$ Las proposiciones serán secretas y se arbitrarán los medios que garanticen tal carácter hasta el momento de la licitación pública, sin perjuicio de lo dispuesto en los artículos 132 y 166 en cuanto a la información que debe facilitarse a los participantes en una subasta electrónica o en un diálogo competitivo.

${ }^{10}$ La procedencia de la aplicación de la prueba de presunciones en el ámbito del procedimiento sancionador ha sido reiteradamente avalada por la doctrina del Tribunal Europeo de Derechos Humanos (Irlanda v. Reino Unido, STEDH 18 enero 1978; Anguelovav. Bulgaria, STEDH 13 junio 2002; Tahsin Acar v. Turquia, STEDH 8 Abril 2004; Nachova y otros v. Bulgaria, STEDH 6 julio 2005; Virabyan v. Armenia, STEDH 2 octubre 2012), del Tribunal Justicia de las Comunidades Europeas (Suiker, STJCE de 16 de diciembre de 1975; Materias Colorantes, STJCE de 14 de julio de 1972; G. Züchner v. Bayerische Vereinsbank AG., STJCE de 14 de julio de 1981; Hi-Fi Pioneer, STJCE de 7 de junio de 1983; Hasselblad, STJCE de 21 de febrero de 1984; Pasta de madera, STJCE de 31 de marzo de 1993), del Tribunal Constitucional (SSTC 174/85 y 175/85, de 17 de diciembre; STC 150/1987, de 1 de octubre; STC 157/98, de 13 de julio) o del Tribunal Supremo (STS de 26 de julio de 1985; STS de 18 de noviembre de 1986; STS de 15 julio de 2002). 
a los interesados la documentación del proyecto. La distribución se hizo en formato $\mathrm{CD}$.

Para ello, el 11 de septiembre de 2008 ArCAIN había entregado a KIROLGINTZAN un CD con la información referente al proyecto de la Instalación «Ola Artificial» (en adelante CD2). En este CD2 constaba el archivo informático denominado XreflCopia de proyectado.dwg. Dicho archivo recogía un plano de cubiertas con cotas detalladas incluidas del que posteriormente debía ser «Complejo Deportivo Pinosolo».

El 2 de octubre de 2008, ARCAIN entregó al Ayuntamiento de Leioa copia en $\mathrm{CD}$ del proyecto de la Instalación «Ola Artificial» (en adelante CD3). En ese CD también consta el archivo denominado informáticamente XreflCopia de proyectado. $d w g$ que contiene el mismo plano.

El plano contenido en los CDs 2 y 3 resultó similar a otro presentado por la Ute Aros-Viuda De SaInz en la licitación del «Complejo Deportivo Pinosolo» en noviembre de 2009 (en adelante CD4).

El CD oficialmente distribuido por KIROLGINTZAN (en adelante CD5) con el proyecto referente a la Instalación «Ola Artificial» es distinto del que ARCAIN le había entregado y no contenía el archivo XreflCopia de proyectado. $d w g$. Ese CD5 fue entregado por KIROLGINTZAN al resto de empresas participantes en la licitación del «Complejo Deportivo Pinosolo»" ${ }^{11}$.

Una comparación visual de los planos presentados en 2008 por ARCAIN (contenido en el archivo XreflCopia de proyectado.dwg del CD2) y en 2009 por la Ute ARos-Viuda De SAINZ (contenido en el CD4) evidencia una similitud tanto con carácter general como en detalles concretos.

En efecto, la similitud general se podía encontrar tanto en zonas generales (piscina, cubiertas, etc.) como en detalles específicos (forma trapezoidal propuesta para las zonas verdes de la entrada en los dos casos, coloreado de la

${ }^{11}$ Dado que los CDs resultaron elementos probatorios relevantes, se incluye una explicación de cada uno de ellos:

- CD1, presentado por DGM en la denuncia;

- CD2, presentado por ARCAIN a KIROLGINTZAN, contiene el proyecto de la Instalación «Ola Artificial» y el archivo «Xref Copia de proyectado.dwg«;

- CD3, presentado por ARCAIN al Ayuntamiento de Leioa, contiene el proyecto de la Instalación «Ola Artificial» y el archivo «Xref $\mid C o p i a$ de proyectado.dwg»;

- CD4, presentado por la Ute Aros-Viuda De Sainz a la licitación del «Complejo Deportivo Pinosolo», contiene el proyecto que constituye su oferta;

- CD5, oficialmente distribuido por KIROLGINTZAN a los licitadores del «Complejo Deportivo Pinosolo», contiene el proyecto de la Instalación «Ola Artificial», pero no contiene el archivo «XreflCopia de proyectado.dwg»;

- CD6, oficialmente distribuido por KIROLGINTZAN a DGM ante notario, una vez realizada la primera adjudicación provisional, contiene el proyecto de la Instalación «Ola Artificial», pero no contiene el archivo «XreflCopia de proyectado.dwg». 
zona de la piscina, etc.). Dada tal similitud visual, se procedió a solicitar sendos informes técnicos.

Así, el informe emitido por el Decano del Colegio Oficial de Arquitectos Vasco Navarro consideró que el plano contenido en el archivo XreflCopia de proyectado. $d w g$ era un encaje volumétrico que incorporaba información muy superior al de un mero esbozo o croquis. Informó, además, que no era, en modo alguno, habitual la fijación de cotas en centímetros en un croquis o encaje volumétrico, a causa del escaso grado de definición que se suele alcanzar en la fase inicial de diseño. Igualmente consideró que no resultaba habitual la fijación de cotas en centímetros en proyectos sometidos a licitación pública. Continuó el informe expresando que las cotas y flechas grafiadas reforzaban el criterio de que el plano examinado formaba parte de un documento con mayor desarrollo que un mero croquis o esbozo, y que en el intento material de ocultamiento de la información disponible, no había sido eliminada la capa de dibujo que contienía dichos datos numéricos y gráficos.

A un requerimiento de información realizado por el SVDC a PUJOL, empresa autora del proyecto presentado por la Ute Aros-ViUda De SAInZ, acerca de si el plano contenido en los CDs 2 y 3 era de su autoría, respondió que, si bien no había sido confeccionado por ella, sí observaba que el diseño de una parte de la cubierta presentaba similitudes con planos confeccionados por ella con ocasión de su trabajo con ARos.

Por todo ello, el CVC consideró probado que el plano presentado por ARCAIN en 2008 al Ayuntamiento de Leioa y a KIROLGINTZAN no era un mero esbozo o croquis sino un documento más elaborado que no podía ser considerado como propio de una fase de preparación de una licitación.

\subsubsection{Similitud de archivos}

En el expediente sancionador instruido a tal efecto, constan varios informes técnicos informáticos que analizan la similitud entre el plano del CD 2 (entregado en 2008 por ARCAIN a KIROLGINTZAN) y el del CD 4 (entregado por la Ute Aros-Viuda De SAInz en 2009en la licitación del «Complejo Deportivo Pinosolo»). Dos de los citados informes fueron encargados por el SVDC y otro fue presentado por DGM junto con la denuncia.

De ellos se derivaron las siguientes constataciones: que en los archivos contenidos en ambos CDs existían capas en las que coincidían partes de los nombres ${ }^{12}$ y que las «cadenas de caracteres» de algunos de los archivos eran coincidentes (H:\PROJECTES-CONCURSOS $\backslash$ Leioa $)$.

${ }_{12}$ EST_PRO, EST_REF, FIP_PRO, FIP_VID, Z_AUX, Z_EDIFICACION, Z_PAV, Z plaza, Z_SIMBOLOGIA, Z_TRA_251, Z_TRA_253, Z_TRA_254, Z_TRA_255, Z_ TXT, Z_VEG, Z_VIAL. 
Ambas constataciones llevaron a conclusiones semejantes. Como expresaba uno de los informes encargados por el SVDC, «estas coincidencias pueden deberse a que uno de los dos archivos parte de una copia del otro, uno de los archivos toma ciertos elementos directamente del otro, o bien en algún momento del proceso ambos han sido editados en el mismo equipo».

Se dio, asimismo, la circunstancia de que, como reconoció ella misma en la instrucción del expediente, la empresa Pujol tenía un antiguo directorio denominado PROJECTES-CONCURSOS, dentro del cual existía un subdirectorio en el que guardaba todo lo relacionado con concursos públicos, incluidos los concursos del Ayuntamiento de Leioa.

\subsubsection{Facturas emitidas por Pujol}

Desde 2008 existían numerosas facturas presentadas por Pujol a ARos en las que el concepto era «Concurso Pinosolo en Leioa. Pago a cuenta de honorarios finales». Las fechas de las citadas facturas eran 23 de julio de 2008, 31 de agosto de 2008, 30 de septiembre de 2008, 30 de octubre de 2008 y 28 de noviembre de 2008. Sin embargo, sólo a partir del 27 de abril de 2011 se formalizó la relación contractual entre Pujol y la Ute AROS-VIUDA De SAINZ.

El 31 de mayo de 2011 la factura presentada a la UTE tenía el concepto de «Nuevo complejo deportivo Pinosolo en Leioa. Gastos de desplazamiento» $\mathrm{y}$, finalmente, el concepto de la factura de 3 de junio de 2011 era «Proyecto básico de arquitectura del complejo deportivo Pinosolo».

La diferencia de cuantías entre las facturas presentadas a Aros antes de la licitación (93.960 euros) y las posteriores a la Ute Aros-ViUda De SAINZ (846.296 euros) es muy significativa.

En consecuencia, la existencia de 5 facturas emitidas por Pujol a Aros previas en más de un año a la publicación de la licitación de 2009 puso de manifiesto, a juicio del CVC, que Aros estaba encargando trabajos relacionados con la licitación desde 2008, antes, por tanto, de la fecha en que se hicieron públicas las características de la licitación.

\subsection{Subsunción de las conductas en el artículo 1.1 de la $L D C^{13}$}

\subsubsection{Existencia de una concertación}

El artículo 1.1 LDC exige la existencia de una concertación que produzca o pueda producir un falseamiento de la competencia en el mercado.

13 «Se prohíbe todo acuerdo, decisión o recomendación colectiva, o práctica concertada o conscientemente paralela, que tenga por objeto, produzca o pueda producir el efecto de impedir, restringir o falsear la competencia en todo o parte del mercado nacional (...)». 
Los hechos probados constituyen, a juicio del CVC, indicios suficientes para deducir que esos hechos encuentran su única razón de ser en la existencia de una concertación consistente en un intercambio de información entre AROS y ARCAIN. En su opinión, la concertación entre AROS y ARCAIN implicó una cooperación entre dos empresas que tuvo como objeto la alteración de las condiciones igualitarias en el proceso de contratación pública del concurso del «Complejo Deportivo Pinosolo». Esa coordinación se inició con el intercambio de información previo a la preparación de los pliegos de la licitación y se prolongó al menos hasta la segunda adjudicación provisional en que intervino ARCAIN.

El CVC tuvo en cuenta que la naturaleza de la concertación que se planteó no era entre empresas directamente competidoras, dado que el papel que jugó ARCAIN en la licitación fue el de empresa consultora del Ayuntamiento de Leioa y de LEIOA KIROLAK. No obstante, este hecho no constituyó obstáculo alguno para que la citada práctica fuera considerada como tipificable en el artículo 1.1, si bien fue tenido en cuenta para determinar la gravedad de la conducta, la cual se consideró grave y no muy grave.

\subsubsection{Existencia de un falseamiento de la competencia en el mercado}

El artículo 1.1 LDC exige, como se ha dicho, la existencia de un falseamiento de la competencia en el mercado. La información intercambiada entre ARCAIN y ARos en el mercado de la licitación era, en opinión del CVC, claramente estratégica y en ningún caso puede resultar equiparable a los contactos que se pueden dar entre licitadores y servicios técnicos y asesores de las entidades públicas, a fin de comprender mejor las características del proyecto licitado. La propia naturaleza de la información intercambiada y el papel que jugaron ambas en la licitación, unido a la relevancia de la garantía del secreto en las licitaciones de concursos públicos, llevaron al CVC a concluir que la concertación tuvo objetivamente aptitud para producir un falseamiento de la competencia que afectó al desarrollo del concurso y por lo tanto al interés público.

En efecto, el intercambio de información previo al concurso del «Complejo Deportivo Pinosolo» invalidó la garantía de no discriminación e igualdad de trato entre los candidatos que establecía el artículo 1 la LCSP ${ }^{14}$. Ade-

14 «La presente Ley tiene por objeto regular la contratación del sector público, a fin de garantizar que la misma se ajusta a los principios de libertad de acceso a las licitaciones, publicidad y transparencia de los procedimientos, y no discriminación e igualdad de trato entre los candidatos, y de asegurar, en conexión con el objetivo de estabilidad presupuestaria y control del gasto, una eficiente utilización de los fondos destinados a la realización de obras, la adquisición de bienes y la contratación de servicios mediante la exigencia de la definición previa de las necesidades a satisfacer, la salvaguarda de la libre competencia y la selección de la oferta económicamente más ventajosa.»» 
más dio al traste con la objetividad y la imparcialidad en la adjudicación. Por todas esas razones, el CVC consideró que se había generado un falseamiento de la libre competencia en ese mercado.

\subsubsection{Conclusión}

El CVC concluyó que el intercambio de información llevado a cabo entre ARCAIN y AROS, empresas no competidoras reales o potenciales en el mismo mercado, pero ambas con capacidad para incidir en el mismo, tuvo por objeto alterar el resultado de la licitación del «Complejo Deportivo Pinosolo» y, por tanto, supuso una infracción del artículo 1.1 de la LDC.

En efecto, el intercambio de información tuvo por objeto falsear, impedir o restringir la competencia en el mercado y objetivamente tuvo aptitud para hacerlo. No fue necesario, por tanto, demostrar la existencia de efectos de dicha conducta. No obstante, quedó acreditado que éstos tuvieron lugar, puesto que ARos ganó la licitación en un proceso viciado en su origen por no haberse respetado las garantías establecidas en la LCSP, habiéndose derivado efectos económicos de tal adjudicación.

\subsection{Subsunción de las conductas en el artículo 3 de la $L D C^{15}$}

De acuerdo con el artículo 3 de la LDC, puede producirse falseamiento de la libre competencia por actos desleales, de los que pueden conocer las autoridades de competencia siempre que los actos afecten al interés público.

La doctrina de la extinta Comisión Nacional de la Competencia al respecto exigía la concurrencia de tres requisitos para poder aplicar el artículo 3 de la $\mathrm{LDC}^{16}$ :

a) La existencia de un comportamiento que pudiera tipificarse como de competencia desleal, para lo cual habría que recurrir a la Ley 3/1991, de 10 de enero, de Competencia Desleal (LCD).

b) Que dicho comportamiento afectase al interés público, esto es, a la libre competencia en el mercado, que aparece configurada como el bien jurídico protegido por la LDC.

c) Que la afectación fuera importante, o lo que es lo mismo, que tuviera entidad suficiente como para causar una grave perturbación en los mecanismos que regulan el funcionamiento del mercado.

15 «La Comisión Nacional de la Competencia o los órganos competentes de las Comunidades Autónomas conocerán en los términos que la presente Ley establece para las conductas prohibidas, de los actos de competencia desleal que por falsear la libre competencia afecten al interés público.»

${ }_{16}$ Véase por todas la Resolución de 9 de octubre de 1991, Expte. A 13/91, SUVECA. 


\subsubsection{El comportamiento de las empresas como acto de competencia desleal}

El artículo 15.2 de la LCD exige para que un comportamiento sea desleal la infracción de las normas jurídicas reguladoras de la actividad concurrencial ${ }^{17}$.

En el caso que nos ocupa, el CVC consideró que la norma reguladora de la actividad concurrencial lo era la LCSP, en concreto en sus artículos $1^{18} \mathrm{y}$ 129. $2^{19}$. La legislación en materia de contratación de las Administraciones públicas constituye regulación de actividad concurrencial, ya que establece limitaciones a la actividad de provisión de bienes y servicios de la Administración en el mercado y, de este modo, establece un marco de igualdad entre los proveedores de bienes y servicios a la Administración.

El art. 129.2 LCSP obligaba a que las proposiciones de los interesados fueran secretas y a que se establecieran los medios que garantizasen tal carácter hasta el momento de la licitación pública. Para garantizar el secreto, las proposiciones se deben remitir en sobres cerrados y sellados. El quebrantamiento de dicho secreto puede afectar al principio de igualdad entre los licitadores en el proceso contractual. Precisamente el carácter secreto de las ofertas en un concurso pretende evitar situaciones de ventaja competitiva. El nivel de detalle en el procedimiento del desarrollo de las licitaciones busca ser garantista con los derechos de los administrados y proteger el interés general permitiendo a la Administración elegir con objetividad la opción más ventajosa.

La exigencia de separación de las distintas fases del procedimiento de licitación (como la proposición y la oferta propiamente dicha) tienen por objeto permitir una evaluación separada de las distintas cuestiones sin condicionar la valoración de cada parte. Todos estos sistemas tienen el objetivo de garantizar el secreto de la oferta en sí. Dicha garantía está puesta al servicio de la igualdad y de la limpieza en los procedimientos de selección. Esta igualdad y limpieza contribuyen a que la adjudicación se otorgue a la oferta más ventajosa $y$, por tanto, más pro-competitiva.

El ilícito de la norma concurrencial (LCSP) llevado a cabo por las partes consistió, a juicio del CVC, en la vulneración del deber de secreto en las proposiciones, considerado probado en base a la prueba de presunciones mencionada. En base a tal prueba de presunciones, el CVC consideró acreditado que ARCAIN (responsable de diseñar los pliegos de prescripciones técni-

17 «Tendrá también la consideración de desleal la simple infracción de normas jurídicas que tengan por objeto la regulación de la actividad concurrencial.»

18 Ver nota 12.

19 Ver nota 7. 
cas del concurso «Complejo Deportivo Pinosolo» desde 2007) tuvo en su poder, casi un año antes de la apertura del concurso, el plano contenido en el archivo denominado XreflCopia de proyectado.dwg que resultó similar y tenía origen común al que en 2009 sería presentado por la UTE AROS-VIUDA DE SAINZ para formular su oferta a la licitación. Además ARCAIN facilitó esa información al Ayuntamiento de Leioia (poder adjudicador en última instancia) antes de la apertura (incluso de la redacción) de los pliegos. Las actuaciones de las empresas rompieron completamente, a juicio del CVC, la garantía de objetividad e imparcialidad en la adjudicación del concurso «Complejo Deportivo Pinosolo», vulnerando la esencia de la fórmula de la licitación pública destinada a garantizar tanto la igualdad de los particulares como la obtención por la convocante, a través de la competencia, de las condiciones más ventajosas para el interés público.

La infracción de la norma concurrencial (LCSP) constituye, además, un acto de competencia desleal subsumible en el párrafo $2^{\circ}$ del artículo 15 de la LCD. Dicha vulneración tuvo efectos negativos sobre el funcionamiento concurrencial del mercado y, en concreto, sobre la posición de igualdad de todas las licitadoras en el Concurso «Complejo Deportivo Pinosolo». Debido a la conducta de las dos empresas, el CVC consideró que no había quedado garantizado que el concurso se hubiera decidido empleando criterios de eficiencia.

\subsubsection{Falseamiento de la competencia}

Para que los hechos puedan ser incardinados en el tipo del artículo 3 de la LDC, resulta necesario que, por la actuación de las empresas, se verifique un falseamiento de la competencia que tenga entidad suficiente como para alterar de forma significativa el desenvolvimiento normal del mercado según la lógica de los hechos económicos. El mercado afectado por el tipo del artículo 3 en relación con una licitación fue considerado por el CVC, de acuerdo con la jurisprudencia, como el de la propia licitación ${ }^{20}$.

El falseamiento se consideró producido porque los actos llevados a cabo por las empresas tuvieron la consecuencia de situar a una empresa en posición de ventaja, en el sentido de que no estuvo asegurado que su selección se hubiera realizado por criterios objetivos. El acceso al mercado y por tanto la competencia para acceder a él se da únicamente en el momento de la licitación. Es, por tanto, la preparación del proceso (en la que se incluye la definición de los pliegos) y la fase de adjudicación (en la que se incluye el proceso

${ }^{20}$ Sentencia de la Audiencia Nacional SAN de 14 de noviembre de 2005, asunto Eléctrica de Eriste, S.L. 
de evaluación de las ofertas) la que permite establecer los diferentes parámetros en los que se generará competencia.

Los elementos que llevaron al CVC a concluir que sí se produjo afectación en el mercado por la competencia se dividieron entre aquéllos que se produjeron durante la preparación de la licitación, en la licitación en sí misma y durante la fase de adjudicación que se extendió hasta la determinación definitiva de la empresa adjudicataria.

Durante la fase de preparación de la licitación, ARCAIN estuvo en posesión, en el momento de la preparación de los pliegos de condiciones técnicas, de un plano similar y que tenía origen común al de una de las empresas que serían licitadoras en el concurso «Complejo Deportivo Pinosolo». Por ello, tuvo la posibilidad, en calidad de asesor cualificado de LEIOA KIROLAK, de influir en los criterios de adjudicación y en la ponderación de cada uno de ellos. De hecho los criterios de adjudicación aprobados por LEIOA KIROLAK otorgaron un número muy elevado de puntos a los siguientes criterios, cuyo cálculo no está sometido a fórmulas matemáticas: $45 \%$ a la propuesta técnica presentada y $10 \%$ al estudio y conocimiento de la obra (siendo las otras, precio, plazo de redacción del proyecto y plazo de ejecución de la obra). Esos criterios son precisamente aquellos donde caben más elementos de subjetividad en el examen de las propuestas.

En la fase de evaluación dos elementos fueron tenidos en cuenta por el CVC. El primero fue que ARCAIN participó en las dos primeras comisiones de evaluación. LEIOA KIROLAK consideró que «no había estado completamente garantizada la imparcialidad y transparencia de la valoración realizada para acordar la adjudicación provisional» y anuló el resultado en dos casos (ante sendos recursos de la denunciante).

Del análisis de la primera adjudicación, el CVC dedujo que habían sido los elementos integrantes de la valoración técnica -en la que participó ARCAIN con dos de los tres votos de la Comisión Evaluadora- los que habían establecido diferencias entre la puntuación obtenida por las distintas empresas licitadoras. La puntuación obtenida por todas las empresas licitadoras en los tres criterios restantes (precio, plazo de redacción y plazo de ejecución) fue idéntica, por lo que, en la práctica, ARCAIN tuvo un peso decisivo en la evaluación.

El CVC no desconoció que existió una tercera Comisión Evaluadora que también consideró que la UTE Aros-ViUda De SAINZ debía recibir más puntuación que las demás, pero este hecho no tuvo entidad como para desvirtuar las conclusiones, teniendo en cuenta todos los elementos previos a la evaluación, los cuales podrían dejar poco margen de actuación a las empresas que participasen en la evaluación posterior.

El segundo elemento tenido en cuenta por el CVC fue que, al seleccionar a una de las licitantes frente a las demás se estaba produciendo una selección 
de la empresa que ocuparía en exclusiva el mercado relevante y se estaba eliminando del mismo a sus competidoras. Si esa selección se realiza sin alterar las reglas de la normativa de contratos y de la normativa de competencia, no merece reproche alguno, dado que la empresa más eficiente es la que ocupa el mercado en aras de la eficacia. En otro caso, la alteración de la competencia resulta evidente, dado que, en el caso examinado, la práctica llevada a cabo implicó que una empresa, prevaliéndose de una práctica ilegal, obtuvo una ventaja competitiva que eliminó a sus competidoras del mercado.

\subsubsection{Afectación al interés público}

El CVC, al examinar la afectación al interés público, tuvo en cuenta que el concurso del «Complejo Deportivo Pinosolo» alcanzó un valor superior a los 50 millones de euros. Pero además, desde un punto de vista cualitativo, consideró que nos encontrábamos ante una licitación pública y que el conocimiento por parte de ArCain del plano de la Ute Aros-Viuda De SAINZ antes de la preparación de los pliegos del concurso era un comportamiento que causó una grave perturbación en el principio de igualdad de trato y libre concurrencia en el mencionado concurso.

Siguiendo la jurisprudencia en materia de competencia, el CVC concluyó que «la afectación del interés público no está ligada al tamaño de la infracción o del mercado en el que se lleva a cabo sino que debe tenerse en cuenta la dimensión del acto de competencia desleal que provoca el falseamiento de la libre competencia ${ }^{21}$.

Por ello, el CVC concluyó que el interés público exige que siempre quede garantizado un proceso concurrencial en el que las empresas capaces de realizar la oferta más ventajosa sean las adjudicatarias de la licitación pública.

\subsubsection{Conclusión}

El CVC concluyó que las actuaciones llevadas a cabo por ARCAIN y ARos constituyeron una infracción del artículo 129.2 de la LCSP por vulneración del deber de secreto. Esa vulneración llevó consigo una vulneración del artículo 15.2 de la LCD en la medida en que se derivó de ella la infracción de normas jurídicas que tienen por objeto la regulación de la actividad concurrencial. Teniendo en cuenta que esa competencia desleal supuso una grave afectación a la competencia y tuvo acreditadas implicaciones de interés público, se generó una infracción del artículo 3 de la LDC.

${ }^{21}$ Íbidem. 


\subsection{Concurrencia de infracciones}

El CVC consideró, en consecuencia, que la conducta llevada a cabo por las empresas ARCAIN y AROs era susceptible de vulnerar no sólo el artículo 1.1 de la LDC, sino también el artículo 3.

La concurrencia de dos ilícitos es clara al analizar los preceptos citados. Los bienes jurídicamente protegidos por ambos preceptos coinciden, dado que en la LDC existe un único bien jurídicamente protegido, que es la libre competencia. Por ello, independientemente de que existan dos infracciones en las que puedan ser subsumibles los hechos probados, y dado que los mismos constituían una unidad, el CVC, para evitar vulnerar el principio non bis in ídem, procedió a imponer una única sanción.

\subsection{Sanción}

El CVC consideró acreditado que Aros y ARCAIN participaron en dos infracciones graves, típicas, antijurídicas y culpables y que su responsabilidad no había prescrito ${ }^{22}$.

Para el cálculo de las sanciones tuvo en cuenta la gravedad de la infracción. El artículo 63.1.b) de la LDC establece que en el caso de las infracciones graves la sanción que puede imponerse puede alcanzar el 5\% del volumen de negocios total de la empresa infractora en el ejercicio inmediatamente anterior al de la imposición de la multa.

En el caso de ARCAIN, dada su labor de consultor externo de la Administración en la licitación pública y dada su capacidad de influencia en la afectación a la competencia, el CVC consideró que existía una agravante muy cualificada, por lo que le impuso una sanción de multa de 89.000 euros.

En el caso de Aros, la sanción de multa impuesta ascendió a 45.000 euros.

${ }^{22}$ Asimismo, consideró que debía absolver a las empresas Pujol y Viuda De SAINZ.

Así, el CVC consideró que no se podía deducir la participación de PuJOL en las conductas constitutivas de infracción del artículo 1.1 LDC ni del 3 LDC.

Con relación a Viuda De SAINZ, el CVC consideró que no se había acreditado su participación en las conductas constitutivas de infracción del artículo 1.1 LDC. Respecto de su participación en la conducta infractora del artículo 3 LDC, el CVC tuvo en cuenta que, si bien formaba parte de la UTE adjudicataria de la licitación del «Complejo Deportivo Pinosolo», había quedado acreditado que ARCAIN tenía en su poder el plano del complejo deportivo al menos desde agosto de 2008, y que el compromiso de constitución de la UTE entre Aros y Viuda De SAInZ, por el contrario, no se produjo hasta el 9 de noviembre de 2009. Por ello, el CVC consideró que su participación en los hechos que constituyen la vulneración del deber de secreto, elemento de la infracción del artículo 3 de la LDC, no había quedado suficientemente acreditada. 
Finalmente, el CVC ordenó a LEIOA KIROLAK la remoción de los efectos de las conductas prohibidas contrarias al interés público. Por tanto, se le ordenó abstenerse de abonar cualquier tipo de indemnización o compensación por daños y perjuicios a las infractoras derivados de la resolución del contrato del «Complejo Deportivo Pinosolo» o, en su caso, recuperar las cantidades ya abonadas en ese concepto.

\section{CONSIDERACIONES FINALES}

Con esta Resolución, el CVC declara de forma indubitada que la contratación pública debe ajustarse a los principios de libertad de acceso a las licitaciones, publicidad y transparencia de los procedimientos, y no discriminación e igualdad de trato entre los candidatos.

A juicio del CVC, el intercambio de información entre una empresa encargada de las labores previas a una licitación pública y otra empresa participante en dicha licitación pública constituye un acuerdo entre no competidores para alterar el resultado de la misma, por lo que supone una infracción del artículo 1.1 de la LDC.

Asimismo, el CVC considera que el citado intercambio de información constituye una infracción de una norma concurrencial, por lo que supone una infracción del artículo 3 de la LDC, en relación con los artículos 15.2 de la LCD y 129.2 de la LCSP.

Por nuestra parte, consideramos que la Resolución de 21 de mayo de 2013 del CVC constituye un elemento jurídico de primer orden que, sin duda, contribuirá a que las administraciones locales de la Comunidad Autónoma de Euskadi interioricen que la normativa reguladora de la libre competencia y la reguladora de la contratación pública están intrínsecamente ligadas, en aras a que las adjudicaciones públicas se produzcan en favor de la empresa que presente la mejor oferta, en favor de una óptima utilización de los recursos públicos y en favor, en consecuencia, del interés público.

TITTLE: Administrative Contracting and Local Administration in the Basque Competition Authority. The 'Pinosolo' Case.

RESUMEN: Se presenta la ocasión de analizar la reciente Resolución de 21 de mayo de 2013 del Consejo Vasco de la Competencia (en adelante, CVC) que resulta sintomática de la necesaria simbiosis entre los entresijos del Derecho de la Competencia y la contratación pública. En esta ocasión se perciben una suerte de prácticas contrarias a la competencia debido a una cooperación entre dos empresas en una licitación pública que tuvieron como objeto la alteración de las condiciones igualitarias en el proceso de contratación pública del concurso del «Complejo Deportivo Pinosolo». De un lado, la concurrencia de la concertación tipificada 
en el artículo 1.1 de la LDC al existir un intercambio de información estratégica entre las empresas. De otro lado, la concurrencia de un acto de falseamiento de la competencia del artículo 3 de la LDC al asistir ante un acto de competencia desleal -en concreto, el del artículo 15.2 de la LCD por infringir las normas reguladoras de la actividad concurrencial (entre otros los artículos 1 y 129.2 de la LCSP) que supone un falseamiento de la competencia al situar ilegítimamente a una empresa en posición de ventaja y que afecta de plano al interés público que descansa en el principio de que la empresa que concurra con la oferta más atractiva sea la adjudicataria de la licitación pública.

PALABRAS CLAVE: licitación pública, imparcialidad, objetividad, concertación, igualdad, falseamiento, interés público, ventaja.

ABSTRACT: The occasion arises to analyze the recent Resolution of May 21, 2013 of the Basque Competition Council (in forward, CVC) that turns out to be symptomatic of necessary symbiosis between the intricacies of the Competition Law and the public contracting. In this occasion there is perceived a kind of practices opposite to the competition due to a cooperation between two companies in a public bidding that took as an object the alteration of the equal conditions in the process of public contracting of the tender of the "Complex Sports Pinosolo». On a one hand, the concurrence of the conciliation typified in the article 1.1 of the LDC due to the existence an exchange of strategic information between the companies. On the other hand, the concurrence of an act of falsification of the competition of the article 3 of the LDC, at an act of disloyal competition -in concrete, that of the article 15.2 of the LCD for infringing the regulatory procedure of the concurrencial activity (among others the articles 1 and 129.2 of the LCSP) that involves a falsification of the competition on having placed illegitimately to a company in position of advantage and that affects flatly to the public interest that rests in the principle of that the company that competes with the most attractive offer is the tenderer of the public bidding.

KEY WORDS: public bidding, impartiality, objectivity, conciliation, equality, falsification, public interest, advantage. 